\title{
On Rhyme, Melody and Artistic Charm of Vocal Music Works in Classical Chinese Poetry
}

\author{
Tong Zeng \\ Guangxi Arts University, Nanning, 530022, China
}

Keywords: Classical Chinese poetry, Artistry, Rhyme, Melody, Artistic charm

\begin{abstract}
Singers must grasp singing skills of multiple styles of vocal music. Deduction of classical Chinese poetry is an important series in multiple styles. To experience artistic charm of vocal music works in classical Chinese poetry, one must deeply understand classical Chinese poetry. Based on summary of long-term teaching experience and practice of singing study, this paper takes works for example to mainly analyze vocal music works in classical Chinese poetry and discuss artistic charm of classical Chinese poetry from several aspects such as literary rhyme and music melody of vocal music works in classical Chinese poetry.
\end{abstract}

\section{Introduction}

China is a country with 5000-year ancient civilization. Classical Chinese poetry as a reprehensive literary form in China runs through historic culture of Chinese nation. In ancient China, classical Chinese poetry was actually an expression of a kind of "songs". So, during reading and chanting poetry, one can feel the lingering charm and experience rhythm of music.

At present, classical Chinese poetry songs generally include two types. The first type includes early ancient art songs or lyrics of Guqin songs, such as Xing Hua Tian Ying created by Jiang Baishi - a composer in the Southern Song Dynasty, Li Xi Mei Ling, Hu Jia Shi Ba Pai, and Yang Guan San Die. The second type includes vocal music works, such as Feng Qiao Ye Bo, Chuan Xiao and Chun Jiang Hua Yue Ye. In order to express rhyme and melody of classical Chinese poetry in the form of modern skills, modern composers absorb modern western composition skills, refer to and study traditional national music styles such as ancient vocal music art, Kunqu opera and Guqin songs, and combine ancient times and present as well as modern times and nation to form study on further creation and deduction of classical Chinese poetry.

\section{Artistry of vocal music works of classical Chinese poetry}

\section{Chanting nature of vocal music works of classical Chinese poetry}

In ancient China, the existence form of classical Chinese poetry is chanting form. Since the Western Zhou Dynasty, people have collected poetry to create music. Vocal music works in classical Chinese poetry form in such way: ancient poetry as the lyrics is composed to music. Rhythm of ancient poetry and music accompaniment make chanting nature of classical Chinese poetry own string lingering charm. Take Xing Hua Tian Ying for example. The lyric was created by Jiang Kui - a litterateur in the Southern Song Dynasty. His lyrics stress melody and concise sentences. His lyrics are quiet, beautiful, quiet, lonely, implicit and far-reaching. In Xing Hua Tian Ying, the author expressed his sadness and depression when travelling along the middle and lower reaches of Yangtze River and passing Nanjing. Flow and plain accompaniment depicts the picture of rover and sky color beside Yangtze River. Skilful application of melody accurately presents the author's mood and the rhythm of the poetry.

\section{Mobility of tune use in vocal music works of classical Chinese poetry}

In vocal music works of classical Chinese poetry, sense of rhythm of music expands in the form of waveform and gradually extends. From single line perspective, waveform expansion of rhythm owns huge development space and can be adjusted flexibly. In music, such creation method is called "run-cavity method". In music, to keep its integrity, the leading position of main sound. Then, 
treatment of derivative sound should focus on main sound. Although this will bring people the feeling that "empty" sound bounces around the main sound, the position of main sound is not changed. The "empty" will form "misty" music rhythm on main sound. The melody of lyrics is visual. The sense of fluctuation forms force in music and emits vigor. Then, the mobility of "run-cavity" is prominent. Take Feng Qiao Ye Bo for example. At the beginning stage of the song, musical instruments are used to imitate the bell tone of a temple which becomes weak from strong. This brings people the feeling from far to near. Thus, people enter the artistic conception of poetry. In terms of vocal music expression, it does not separate from poetry rhythm, but vocal music is created on the basis of poetry rhythm. Seeing from vocal music creation, it is based on rhyme of ancient poetry. Seeing from singings method of music, prolongation of tune is reflected in the last word of verses. Not just does intonation comply with reading methods of classical Chinese poetry, but also unique lingering charm of classical Chinese poetry can reflect.

Ancient poetry is very rich in sense of rhythm, but monotony still exists. In order to get rid of linearity effect and form flowing music in vocal music works of ancient poetry, flexible "run-cavity" is required to make ancient poetry expressed by music embody powerful tension and generate mobility. This should be specially stressed during deducing vocal music works of ancient poetry.

\section{Rhyme beauty of vocal music works in classical Chinese poetry}

As for communication and development of poetry, an important cause is that music factor exists in it and fusion of poetry and music mood expression is achieved to gain beautiful artistic state. Music expresses sound. Poetry is a visual realm. The combination of the two forms poetry evolution history. Folk songs from Tan Ge in ancient times to The Book of Songs, The Songs of Chu in the Warring States Period and seven-word lyrics in Han-Wei Period as well as collection of poetry in Han Dynasty impose great influence on advanced music works of ancient poetry. With regard to rhythm of classical Chinese poetry, due to influence of writing methods, one can experience metaphor and analogy means which highlight chanting nature of poetry from The Book of Songs. During pronunciation in singing, one should know how to use breath. For instance, slow pronunciation method of poetry chanting requires the sound of the first word should be extended. The essential vowel in a compound vowel and the tail of a word should be ended quickly to return the rhyme.

In Chinese literature masterpiece Wen Xin Diao Long, the creator Liu Xie proposed that, "poetry is the heart of music; sound is the body; the music body lies in sound; blind musicians should adjust their music instruments; the core of music lies in poetry; gentlemen should write poetry well”. The close relationship between poetry and music is explicit. Poetry and lyrics should be mutually related and keep respective independence to achieve fusion of the two. In modern vocal music works of ancient poetry, rhyme is reflected, which is not limited to the form. Then, when creators are composing, they should respect expression of poetry, know core of poetry and understand mental state of poetry creators.

\section{Melody beauty of vocal music works in classical Chinese poetry}

Vocal music works in classical Chinese poetry stem from songs of classical Chinese poetry and own a history of over 3000 years. Vocal music in classical Chinese poetry and the origin of music are closely related. As the treasure of Chinese traditional culture, the language of vocal music works is concise and they have rich story plots. Besides, the emotion is presented through rhythm per second and continues till now with historic culture. Till now, vocal music works of ancient poetry are the study objects of numerous experts. And, singers prefer to them. Vocal music works of classical Chinese poetry apply creation means of modern music. Ancient poetry serves as lyrics. Modern melody is applied to reach perfect combination of lyrics and melody and combination of ancient and present melody and lyrics. Plus effects of rhythm, atmosphere of poetry is rendered. The audiences show fluctuated moods under the fusion of feelings with the natural setting. Since ancient poetry contains rich feelings of ancient people and abundant ideological implication, they can better make 
audiences experience the lingering charm after they are treated to vocal music works. Chang Xiang Zhi is a vocal music works with melody beauty in classical Chinese poetry. It is expressed with graceful tone and melody. Te language is used to render artistic conception. Under dulcet melody, it presents classical lingering charm. In particular, music adopts tune of Kunqu opera to extend. The melody shown with appoggiatura and bladder sound further presents the beauty of melody.

\section{Rhythm beauty of Chang Xiang Zhi}

"Rhythm" belongs to aesthetic category. Its rich contents are not limited to aesthetic category realm expressed by literary works. They generate emotional resonance with audiences. Aesthetic appreciation expressed with "rhythm" mainly refers to a feeling established by use of the correlation between subject and object. Both performers and audiences can feel far-reaching deduction and beauty of artistic conception. In Chinese classical aesthetics, "rhythm" is kind of aesthetic experience which is stressed in each historical period. In different periods, there is diverse positioning for "rhythm" beauty. What presents is expression of unique national style.

Some believe the highest beautiful realm of classical Chinese poetry is the realm of sound as the flower and argumentation as the fruit and express with language rhythm. Such beauty of lingering charm is just an important norm of Chinese vocal music works. For national vocal music works, the beauty of "rhythm" mainly applies song language to express emotions. Sound realization, melody change, well-matched words and harmonious tone give full play to the beauty of "rhythm". In Chang Xiang Zhi based on ancient poetry, three main aspects of rhythm (level and oblique tones, rhyme and antithesis) are applied skillfully, and the express is proper and natural. Thus, the whole lyric of Chang Xiang Zhi brings people euphemistic and sincere sense of beauty by virtue of its archaic Chinese rhyme. Meanwhile, it also contains primitive simplicity and delicateness. The heroine's faithful love for the hero is fully expressed. The lyric expression of Chang Xiang Zhi continues proper metaphor and analogy methods in classical Chinese poetry. The lasting and deep love story is narrated quietly with the music. Dense romantic theme is shown.

\section{Rhythm beauty of tune of Chang Xiang Zhi}

Although Chang Xiang Zhi expresses love manifesto and language is bold and straight-forward, the tune is not single and can bring people the soul-stirring feeling. Seeing from the whole vocal music work, the tune will bring people the feeling of continuing for ever. As for rhythm beauty, grace note is mainly applied to express euphemistic and long-lasting tune. In the whole works, grace note can be seen everywhere, but does not lose the beauty of archaic Chinese rhyme. In particular, the application of small appoggiatura and trill appropriately expresses artistic conception of the poetry. Both the motion expressed in language and emotional flexibility in tone bring people the different feeling. Under the company of grace note, the singer should accurately express the protagonist's emotion, which is crucial.

Chang Xiang Zhi mainly refers to singing method of Kunqu opera. During singing Kunqu opera, appoggiatura, major second or major third should be added when falling tone is met. But in the fourth tone, appoggiatura should be applied behind it. During singing, it is necessary to first pause and then use the tune. The explanation of singing method of Kunqu opera is recorded and narrated in $G u W u$ Lu Si Sheng Ji Lue, "for the fourth tone, it is required to avoid chanting in the slow, continuity and immediately stop during the opening of mouth; for the song with close rhythm, it is required to immediately stop during the opening of mouth, without any delay". When this singing method is applied for Chang Xiang Zhi, the breath should be controlled at the beginning and it should be sung directly without turning. When the sound will rise, it is required to slightly pause. The rising force should become strong from weak. To reflect lingering charm of drama singing method, prolongation of tune should be used. The excellence of Chinese drama is that the long-lasting tune can being people the feeling of ups and downs, just like emotional ups and downs, while music lingering makes one own endless aftertastes.

In Chang Xiang Zhi, small appoggiatura is a major method which runs through the whole music. When small appoggiatura appears, appreciators will feel as if they enter Han Dynasty and enjoy the music beauty. For example, the lyric "I will love you till the end of my life" skillfully applies small appoggiatura. The mood of the heroine is exactly expressed. Besides, the beauty of heroine is fully 
shown. In the whole vocal music works, small appoggiatura is full of it so that the whole song is integrated and natural.

In Chang Xiang Zhi, the rhythm beauty is also reflected in tail sound treatment. Seeing from singing skills of Kunqu opera, the sound extends on one word and then the next word is sung. Such singing method is especially obvious on the tail sound. In Chang Xiang Zhi, especially the tail sound part, almost every word adopts the method of one word with multitone so that the song sounds like narration of a story and emotional narration. The whole song expresses deep feeling. Although the lyric of Chang Xiang Zhi is straight-forward, mitigatory and fluctuant composition presents melody of classical Chinese poetry. Slow pronunciation, soft tune and music range become weak from strong. Trill is interspersed to fully show long-lasting classical lingering charm.

\section{Conclusions}

To sum up, in current multicultural society, music art of ancient poetry is active in art stage by virtue of its exuberant life. Only when one understands special rhyme and melody of ancient poetry and combines sound production skills with comprehension can deduction of ancient poetry fully show unique charm which is reflected through close combination of literature and art and profound artistic conception of Chinese ancient culture. Hence, the study on vocal music works of classical Chinese poetry not just contributes to deep understanding of Chinese excellent culture, but also expands broader prospect for inheriting and developing musical culture of classical Chinese poetry.

\section{References}

[1] Liu Yu, On expression of artistic conception of art songs of classical Chinese poetry in vocal music performance. Journal of Nanchang College of Education, 2011.26 (09)

[2] Wu Yue, On artistic characteristics and singing of vocal music works recomposed with ancient poetry. Time Education, 2010(03)

[3] Guo Kejian, Singing methods - theory and practice of China's vocal music art . Beijing: Culture and Art Publishing House, 2007

[4] Niu Yueying, Singing style of classical poems and Chinese art songs. Journal of Shenyang Normal University (Social Science Edition), 2012.36 (03)

[5] Wang Xuemei, Fuse poetry and music and bring out the best in each other - on fusion relationship between music and poetry. Journal of Shaoyang University (Natural Science Edition), 2004.3 (04)

[6] Li Fangyi, Profound literary art - discussion on characteristics and singing of art songs of classical Chinese poetry. The Academic Periodical of Shenyang Conservatory of Music, 2010 (03)

[7] Sun Pain, Archaic Chinese rhyme - discussion on characteristics and singing of art songs of classical Chinese poetry. Heilongjiang: Editorial Department of Youth Litterateurs, 2011 (21) 